\title{
Can herbal medicinal products or preparations alleviate neuropathic pain in adults? A Cochrane Review summary with commentary
}

\author{
Chiara Arienti* \\ IRCCS Fondazione Don Carlo Gnocchi, Milan, Italy
}

\begin{abstract}
.
BACKGROUND: Several medicines have been shown to be effective in treating neuropathic pain, but they can have damaging side effects, therefore people are now trying herbal products to help relieve pain.

OBJECTIVE: The aim of the Cochrane Review was to evaluate whether herbal products/preparations have an analgesic effectiveness in neuropathic pain and whether any side effects are associated with their use.

METHODS: The population addressed were adults aged $\geq 18$ years, suffering from chronic neuropathic pain. Studies that investigated the effects of herbal products/preparations for the relief of neuropathic pain compared to any or no interventions were included.

RESULTS: Included studies did not show that herbal products/preparations have reduced pain intensity of $30 \%$ or above and there was no observable reduction in the total pain score.

CONCLUSIONS: There is insufficient evidence to suggest that herbal products/preparations have any efficacy in any neuropathic pain conditions. The current evidence is of very low quality resulting in serious uncertainties about the estimates of effect observed.
\end{abstract}

Keywords: Herbal medicine, neuropathic pain, rehabilitation

The aim of this commentary is to discuss in a rehabilitation perspective the published Cochrane Review "Herbal medicinal products or preparations for neuropathic pain" (Boyd et al., 2019) by Boyd et al. ${ }^{\text {a }}$, under the direct supervision of Cochrane Pain, Palliative and Supportive Care Group. This Cochrane

*Address for correspondence: Chiara Arienti, IRCCS Fondazione Don Carlo Gnocchi, Via Alfonso Capecelatro 66, 20148, Milan, Italy. Tel.: +39 0307245282; E-mail: carienti@dongno cchi.it.
Corner is produced in agreement with NeuroRehabilitation by Cochrane Rehabilitation.

\footnotetext{
${ }^{a}$ This summary is based on a Cochrane Review previously published in the Cochrane Database of Systematic Reviews 2019, Issue 4, Art. No.: CD010528, DOI: 10.1002/14651858.CD010528. pub4 (see www.cochranelibrary.com for information). Cochrane Reviews are regularly updated as new evidence emerges and in response to feedback, and Cochrane Database of Systematic Reviews should be consulted for the most recent version of the review.

The views expressed in the summary with commentary are those of the Cochrane Corner author and do not represent the Cochrane Library or Wiley.
} 


\section{Background}

The management of chronic neuropathic pain is challenging and is best achieved within a multidisciplinary team. Pain is a subjective experience, and it is important to validate a patient's pain, address psychosocial comorbidities, and set realistic treatment goals. Evidence-based guidelines are available to guide treatment, but frequently, highquality evidence-based recommendations are lacking (Zilliox, 2017). Neuropathic pain is a frequent condition that is often resistant to treatment and is associated with poor patient satisfaction of their treatment. Several medicines have been shown to be effective in treating neuropathic pain associated with diabetic neuropathy and postherpetic neuralgia, and these medicines are often used to treat neuropathic pain associated with other conditions as well. These medicines can have damaging side effects, therefore people are now trying herbal products/preparations to help relieve pain instead (Meng et al., 2017).

A Cochrane systematic review evaluated whether herbal medicinal products/preparations have an analgesic effect and their effectiveness for neuropathic pain and whether any side effects are associated with their use (Boyd et al., 2019).Herbal medicinal products or preparations for neuropathic pain (Boyd et al, 2019)

\section{Objective}

The aim of this Cochrane systematic review was to assess the analgesic efficacy and effectiveness of herbal medicinal products/preparations for neuropathic pain, and the adverse events associated with their use.

\subsection{What was studied and methods}

The population addressed were adults aged $\geq 18$ years, suffering from one or more neuropathic pain conditions, for three months or more. Neuropathic pain conditions included (but were not limited to) the following: painful diabetic neuropathy (PDN); post-herpetic neuralgia (PHN); trigeminal neuralgia; phantom limb pain; postoperative or traumatic neuropathic pain; complex regional pain syndrome (CRPS); cancer-related neuropathy; HIV neuropa- thy; spinal cord injury. There were no restrictions based on gender.

Studies that investigated the effects of herbal medicinal products/preparations administered in the form of whole plants, parts of plants or extracts for the relief of neuropathic pain compared to placebo, no intervention or any other active comparator were included. Studies monitoring other analgesic consumption, alongside herbal medicinal products were also included.

Studies monitoring the effects of isolated, single chemicals derived from the plant or synthetic chemicals based on constituents of the plant, monitoring the effects of traditional Asian medicine and monitoring the effects of capsaicin or cannabis were excluded. Studies of headache or migraine were also excluded.

Primary outcomes studied were pain relief of $30 \%$ or greater and of $50 \%$ or greater, and the participant-reported global impression of clinical change (PGIC) much or very much improved (moderate or substantial). Secondary outcomes studied were: any pain-related outcome indicating some improvement; withdrawals for any reason and adverse events, including death.

\section{Results}

The review included 2 studies with 128 participants, in total, 91 of whom were treated with the herbal interventions (nutmeg and St John's wort), in comparison with placebo. One study $(\mathrm{N}=74)$ included only participants suffering from diabetic neuropathy (Motilal 2013). Another study $(\mathrm{N}=54)$ included participants with diabetic and non-diabetic neuropathic pain conditions, including those with polyneuropathy and idiopathic peripheral neuropathy (Sindrup 2000). Participants' age range was 21 to 85 years. Both studies included men and women.

Due to heterogeneity in primary outcomes and failure of study authors to provide requested data, meta-analysis was not performed. The evidence derived from this review was downgraded to very low quality due to limitations in study quality and imprecision. Low study quality was attributed to various factors such as study size, attrition bias, short duration of intervention and followup. For this reason, subgroup analysis was not carried out.

The results of the review showed the following: 


\subsection{Participant-reported pain relief of $30 \%$ or greater over baseline (moderate)}

One study reported a participant-reported pain relief of $30 \%$ or above over baseline, in response to nutmeg versus placebo (RR 1.12, 95\% CI 0.69 to 1.85 ; $48.6 \%$ vs $43.2 \%$; Motilal 2013). Participantreported pain relief of $30 \%$ or greater over baseline is a moderate effect as described by the Initiative on Methods, Measurement, and Pain Assessment in Clinical Trials (IMMPACT) definitions of moderate, however, there was no significant difference between the two groups. The quality of evidence was downgraded by three levels to very low due to very serious limitations in study quality (small participant numbers and attrition bias) and indirectness (short-term outcomes only).

\subsection{Participant-reported pain relief of $50 \%$ or greater, over baseline (substantial)}

Neither study reported substantial pain relief of $50 \%$ or greater.

\subsection{Participant-reported global impression of clinical change (PGIC) much or very much improved (moderate)}

Neither study reported PGIC much or very much improved.

\subsection{Participant-reported global impression of clinical change (PGIC) very much improved (substantial)}

Neither study reported PGIC to be very much improved.

\subsection{Any pain-related outcome indicating some improvement}

The study of Motilal 2013 revealed no change between placebo ( $44 \pm 21.5)$ and nutmeg $(44 \pm 26.5)$ treatments in pain relief evaluated with Visual Analogical Scale (VAS). The study of Sindrup (2000) demonstrated a reduction of 1 point from baseline at weeks two to five on a 0 to 10 -point numeric rating scale. This small change can be considered as no evidence of change between the two groups.

\subsection{Withdrawals}

The two studies observed five withdrawals out of 91 participants $(5 \%)$ in the treatment groups compared to six withdrawals out of 91 participants $(6.5 \%)$ in the placebo groups, giving an increased RR for withdrawal with active treatment (RR $0.83,95 \%$ CI 0.26 to 2.64 ; $\mathrm{NNTH}=1.7$ ).

\subsection{Adverse events}

The two studies reported a total of 17 adverse events in the treatment groups (nutmeg and St John's wort). The combination of the two studies showed a RR of 1.00 (95\% CI 0.55 to 1.81 ; NNTH = 10; Analysis 2.1), for adverse events in response to these herbal treatments, and an odds ratio of 1.00 (95\% CI 0.47 to 2.15). The quality of the evidence for this outcome was downgraded to very low as a result of very serious limitations in study quality and also indirectness.

Neither study reported any deaths or serious adverse events.

\section{Conclusions}

The authors concluded that there is insufficient evidence to suggest that nutmeg or St. John's wort has any efficacy in analyzed neuropathic pain conditions. The current evidence is of very low quality resulting in serious uncertainties about the estimates of effect observed. The evidence on adverse events is very low quality and therefore caution should be applied to its usage until more research has been done in this area.

\subsection{Implications for practice in neurorehabilitation}

The Cochrane Review (Boyd et al., 2019) summarized in this Cochrane Corner Rehabilitation synthesizes the possible effectiveness of herbal medicinal products or preparations administered in the form of whole plants, parts of plants or extracts for the relief of neuropathic pain. Only two small studies were found, which analyzed limited number of herbal medicinal products, consequently, there is uncertainty or very low certainty of evidence on their efficacy and on the adverse events that they can produce.

Rehabilitation is a set of interventions designed to optimize functioning and reduce disability in individuals with health conditions in interaction with 
their environment and rehabilitation professionals are caring for individuals with various different health conditions that often include the treatments of neuropathic pain to improve the quality of life.

This Cochrane Review suggests to rehabilitation professionals to be careful when considering the use of herbal interventions in people suffering from neuropathic pain and to continue offering the standard care for its management.

\section{Acknowledgments}

The author thanks Cochrane Rehabilitation and Cochrane Pain, Palliative and Supportive Care Group for reviewing the contents of the Cochrane Corner.

\section{References}

Boyd, A., Bleakley, C., Hurley, D. A., Gill, C., HannonFletcher, M., Bell, P., \& McDonough, S. (2019). Herbal medicinal products or preparations for neuropathic pain. The Cochrane Database of Systematic Reviews, 4, CD010528. http://dx.doi.org/10.1002/14651858.CD010528.pub4.

Meng, H., Johnston, B., Englesakis, M., Moulin, D. E., \& Bhatia, A. (2017). Selective Cannabinoids for Chronic Neuropathic Pain: A Systematic Review and Meta-analysis. Anesthesia and Analgesia, 125(5), 1638-1652. https:// doi.org/10.1213/ANE.0000000000002110.

Zilliox, L. A. (2017). Neuropathic Pain. Continuum (Minneapolis, Minn.), 23(2, Selected Topics in Outpatient Neurology), 512-532. https://doi.org/10.1212/CON.0000000000 000462 .

\section{Conflict of interest}

The author declares no conflicts of interest. 